\title{
Electrolyte abnormalities in diabetes mellitus in the presence of normal serum creatinine
}

\author{
Tahniyah Haq ${ }^{1}$, Shapur Ikhtaire², Mehruba Alam Ananna³, Shohael Mahmud Arafat ${ }^{2}$
}

${ }^{1}$ Department of Endocrinology, Bangabandhu Sheikh Mujib Medical University (BSMMU), Dhaka, Bangladesh.

${ }^{2}$ Department of Medicine, BSMMU, Dhaka, Bangladesh.

32Department of Nephrology, Bangladesh Institute of Research, Rehabilitation of Diabetes, Endocrine and Metabolic Disorders

(BIRDEM), Dhaka, Bangladesh.

Abstract

Aim: Diabetes mellitus is a metabolic disorder that causes disturbances in the electrolyte regulating mechanisms of our body. The purpose of the study was to determine the pattern of electrolyte imbalance in patients with diabetes mellitus and compare with healthy individuals; and to correlate the degree of electrolyte imbalance with control and duration of diabetes.

Methods: This cross-sectional study was conducted over a period of 6 months. It included 90 cases with diabetes mellitus and 50 cases without diabetes mellitus. All subjects had normal serum creatinine level. Serum electrolytes (sodium, potassium, chloride and bicarbonate) were measured in each subject by automated methods. The normal levels of sodium, potassium, chloride and bicarbonate were taken as 136-146, 3.5-5, 102-109 and 22-30 mmol/L respectively. Levels above or below this reference were taken as abnormal. Control of diabetes was determined by measuring HbA1c using high-performance liquid chromatography.

Result: The most common abnormalities in patients with diabetes mellitus were hypochloraemia (48.89\%), low serum bicarbonate $(23.33 \%)$ and hyponatraemia $(11.11 \%)$, though the mean values of serum electrolytes were within the normal reference range. Serum bicarbonate level was significantly lower in the group with diabetes mellitus $(p=0.010)$. There were a significantly greater number of cases with hyponatraemia and hypochloraemia among cases with diabetes mellitus $(p<0.016)$. Serum sodium was negatively correlated with glycaemic control $(\mathrm{r}=-0.39 ; \mathrm{p}<0.001)$ and duration of diabetes $(\mathrm{r}=-0.31 ; \mathrm{p}=$ $0.003)$.

Conclusion: Electrolyte abnormalities are more common in patients with diabetes mellitus even with normal serum creatinine, and this correlates with control and duration of diabetes. (SJDEM 2020/ Vol 10/No 2)

Keywords: Electrolyte, hyponatraemia, hypochloraemia, bicarbonate, type 2 diabetes mellitus

Correspondence email: tahniyah81@bsmmu.edu.bd

ORCID ID: https://orcid.org/0000-0002-0863-0619

Copyright: This is an open-access article distributed under the terms of the Creative Commons Attribution License, which permits unrestricted use, distribution, and reproduction in any medium, provided the original author and source are credited (CC BY 4.0)

\section{Introduction}

There is considerable morbidity among diabetic patients. About $30 \%$ of patients with type 2 diabetes mellitus have nephropathy. Diabetic kidney disease is defined as gradually increasing urinary albumin excretion and blood pressure (1). Less attention is given to tubular function. The tubules are engaged in maintaining of the body's electrolyte. Therefore, a tubular dysfunction results in electrolyte abnormality. It is not known when tubular dysfunction begins in type 2 diabetic cases. This important,as nephropathy is associated with other microvascular complication.

There have been several studies on electrolyte disorder in patients with diabetes mellitus. A study in Greece stated that acid-base and electrolyte disorders often occurred in diabetic patients even if the renal function was normal. Patients that had serum creatinine $<107 \mu \mathrm{mol} / \mathrm{L}$ had metabolic alkalosis and hypernatraemia (2). A study was carried out in Pakistan to evaluate the electrolyte homeostasis during the progression of various stages of diabetic nephropathy. Serum sodium level was decreased in 
diabetic, diabetic hypertensive and diabetic nephropathy patients. The activity of $\mathrm{Na}+\mathrm{K}+$-ATPase was also significantly reduced in all groups3. Patients with type 1 diabetes mellitus had a significant increase in the level of sodium and little or no corresponding increment in the potassium concentration(4). Oyewole et al. showed a significant reduction in serum potassium, sodium and bicarbonate and an elevation of chloride among patients with type 2 diabetes mellitus(5). The reduction may be the result of electrolyte loss caused by kidney dysfunction6. Another study also demonstrated significant reduction in serum sodium level among type 1 and 2 diabetic patients, especially among insulin treated patients. There was no significant change in serum potassium(7). Other studies found raised sodium, potassium and chloride levels in people with diabetes, irrespective of renal function ${ }^{(8,9)}$. Compared to healthy individuals, serum potassium was significantly increased and serum magnesium significantly decreased in patients with diabetes mellitus ${ }^{(10)}$. Electrolyte abnormalities were seen in hospitalized patients with diabetes mellitus, irrespective of renal function. Hyponatraemia was the most common electrolyte imbalance $(80 \%)$, followed by hypomagnaesemia (38\%), hypokalaemia $(36 \%)$ and hyperkalaemia $(14 \%)$. The precipitating factors were vomiting, drugs, diarrhea and renal failure ${ }^{(11)}$

Variable patterns of electrolyte abnormalities occur in diabetes mellitus due to a multitude of factors. The reduction in serum sodium and potassium might be a result of electrolyte loss through the kidneys (6). Another cause of this electrolyte imbalance might be due to inhibition of the rennin-angiotensin-aldosterone system (12). The low serum insulin level in patients with diabetes leads to lack of inhibition of ketogenesis. In this condition, ketone bodies in the blood are elevated with elevated blood bicarbonate level and resultant metabolic acidosis (13). The reduction in blood $\mathrm{pH}$ caused by ketoacidosis might result in acid base imbalance which may lead to elevation of chloride by the system in order to compensate for the anion loss (14). Both hypo and hypernatremia, resulting from hyperglycemia related mechanisms are seen in diabetes. Chronic hyperkalemia results from hyporeninemic hypoaldosteronism, impaired renal function, use of potassium-sparing diuretics, hypertonicity and insulin deficiency ${ }^{(15)}$.

There is some evidence from previous studies that electrolyte imbalance occurs in diabetes mellitus with normal serum creatinine. This may be due to renal abnormalities or problems in other homeostatic mechanism. Early detection and treatment to correct the electrolyte imbalance would bring about better outcome for patients.

\section{Objective}

This study was undertaken to determine the pattern of electrolyte abnormalities in diabetes mellitus with normal creatinine level and compare it with healthy individuals; and to correlate the degree of electrolyte imbalance with control and duration of diabetes mellitus.

\section{Method}

Design

This 6 month study was carried out in Bangladesh Institute of Research and Rehabilitation in Diabetes, Endocrine and Metabolic Disorders (BIRDEM) hospital. Patients with diarrhea, vomiting, diabetic ketoacidosis, hyperosmolar hyperglycaemic state, Addison's disease, Cushing's syndrome, heart failure, chronic liver disease, nephrotic syndrome and those on diuretics, angiotensin converting enzyme inhibitors and angiotensin receptor blockers were excluded.

The study included 90 adult patients with previously diagnosed diabetes mellitus (group 1) and 50 adults without diabetes mellitus (group 2). All 140 participants had normal serum creatinine level. Serum electrolytes were measured in each group by automated methods. HbA1c was measured in group 1 . The study was approved by the Ethical Review Committee of Diabetic Association of Bangladesh (BADAS).

\section{Study procedure and operational definition}

Medication history was taken from each participant with a predesigned questionnaire. Height, weight and blood pressure were measured systematically using stadiometer, calibrated scale and Yamasu aneroid sphygmomanometer. Serum sodium, potassium, chloride and bicarbonate were measured by Nova 8 Analyzer using the principle of ionic diffusion with ion selective electrodes as measuring technology. Serum creatinine was measured by Siemens Dimension clinical chemistry system. The Chronic Kidney Disease Epidemiology Collaboration (CKD-EPI) equation was used to calculate eGFR. The equation used was eGFR $=141 * \min (\mathrm{Scr} / x, 1) \alpha * \max (\mathrm{Scr} / x, 1)-1.209 * 0.993 \mathrm{Age} *$ 1.018 [if female] * 1.159 [if black] 16 . HbA1c was estimated on the principles of ion-exchange high-performance liquid chromatography (HPLC) by BIO-RAD Variant II Turbo analyzer. Urinary albumin and creatinine were measured by Atellica CH 930 analyzer. Albumin creatinine ratio (ACR) was calculated by the following formula - urinary albumin/urine creatinine. Proteinuria was detected by dipstick method at the bedside. The normal levels of sodium, potassium, chloride and bicarbonate were 136-146, $3.5-5,102-109$ and $22-30 \mathrm{mmol} / \mathrm{L}$ respectively ${ }^{(17)}$.

\section{Data analysis}

Data were analyzed by SPSS version 11.5. The mean values of serum electrolytes were expressed as mean \pm SD. Frequencies of serum electrolyte abnormalities were determined at 95\% confidence interval. Unpaired t test and Chi square test were used to compare cases with and without diabetes mellitus. Partial correlation coefficient test and linear regression was done between serum electrolytes and $\mathrm{HbA} 1 \mathrm{c}$, diabetes duration, eGFR. P value of $\leq 0.05$ was considered significant. ResultsTable 1 and 2 shows the clinical conditions of group $1.97 .78 \%$ patients in group 1 had eGFR above $60 \mathrm{ml} / \mathrm{mim} / 1.73 \mathrm{~m} 2$. eGFR of the other 2 patients was $57 \mathrm{ml} / \mathrm{mim} / 1.73 \mathrm{~m} 2$. 
Table 1 Clinical and laboratory parameters of patients with diabetes mellitus/group $1(n=90)$

\begin{tabular}{lll}
\hline Variable & Mean \pm SD & Range \\
\hline Age (years) & $50.92 \pm 11.33$ & $27-75$ \\
Duration of diabetes (yrs) & $5.20 \pm 5.66$ & $0-20$ \\
BMI (kg/m2) & $27.16 \pm 4.72$ & $17-47$ \\
Systolic Blood Pressure (mmHg) & $123.45 \pm 15.11$ & $100-170$ \\
Diastolic Blood Pressure (mmHg) & $79.83 \pm 8.82$ & $60-100$ \\
HbA1c (\%) & $8.62 \pm 2.36$ & $5-15$ \\
Urine Micro albumin (mg/L) & $14.46 \pm 17.53$ & $5-117$ \\
Albumin Creatinine Ratio (mg/g) & $24.08 \pm 20.84$ & $11-181$ \\
eGFR (ml/mim/1.73m2) & $95.12 \pm 20.57$ & $57-152$ \\
\hline
\end{tabular}

Table 2 Frequencies of clinical conditions of patients with diabetes mellitus/group $1(n=90)$

\begin{tabular}{lll}
\hline Clinical condition & Number & Percentage \\
\hline Treated with diet and exercise & 38 & 42.22 \\
Treated with oral anti-diabetic drugs & 35 & 38.89 \\
Treated with insulin & 15 & 16.67 \\
Overweight $(\geq 25 \mathrm{~kg} / \mathrm{m} 2)$ & 59 & 65.56 \\
Hypertension & 23 & 25.56 \\
Systolic $>140 \mathrm{mmHg}$ ) & 10 & 11.11 \\
Diastolic $(>90 \mathrm{mmHg})$ & 6 & 6.67 \\
On anti-hypertensive drugs & 13 & 14.44 \\
Uncontrolled diabetes (HbA1c $>7 \%)$ & 63 & 70 \\
Albuminuria (U. microalbumin $>30 \mathrm{mg} / \mathrm{L})$ & 6 & 6.67 \\
\hline
\end{tabular}

Electrolyte abnormality in the study population

The mean values of individual electrolytes in cases with diabetes mellitus were within the normal range (Table 3). However some cases of electrolyte abnormality were found. The most common electrolyte abnormalities were hypochloraemia $(48.89 \%)$, raised serum bicarbonate level $(23.33 \%)$ and hyponatraemia $(11.11 \%)$ (Table 4$)$. 


\begin{tabular}{lccc}
\hline Variable & Cases with diabetes & Cases without diabetes & P value \\
& $\begin{array}{c}\text { mellitus } \\
(\mathbf{n}=\mathbf{9 0})\end{array}$ & mellitus \\
& Mean \pm SD & $(\mathbf{n}=\mathbf{5 0})$ & \\
& $27.90 \pm 3.44$ & Mean \pm SD & $0.01^{*}$ \\
\hline Serum Bicarbonate (mmol/L) & $139.19 \pm 3.78$ & $28.20 \pm 2.10$ & 0.09 \\
Serum Sodium (mmol/L) & $4.29 \pm 0.40$ & $141.18 \pm 2.04$ & 0.14 \\
Serum Potassium (mmol/L) & $101.66 \pm 5.24$ & $4.25 \pm 0.31$ & 0.17 \\
Serum Chloride $(\mathrm{mmol} / \mathrm{L})$ & $103.02 \pm 2.68$ & \\
\hline
\end{tabular}

Note: *Significant; student's independent t test.

\section{Table 4 Electrolyte abnormalities in the study population $(n=140)$}

\begin{tabular}{lccc}
\hline Electrolyte & Cases with diabetes & Cases without diabetes & P value \\
abnormalities & mellitus & mellitus & \\
& $\mathbf{( n = 9 0 )}(\mathbf{0})$ & $\mathbf{( n = 5 0 )}$ & \\
\hline Hyponatraemia & $10(11.11)$ & $\mathbf{n}(\mathbf{\%})$ & $0.01^{*}$ \\
Hypernatraemia & $2(2.22)$ & $0(0)$ & 0.29 \\
Hypokalemia & $2(2.22)$ & $0(0)$ & 0.29 \\
Hyperkalaemia & $1(1.11)$ & $0(0)$ & 0.65 \\
Hypochloraemia & $44(48.89)$ & $3(6)$ & $0.02 *$ \\
Hyperchloraemia & $2(2.22)$ & $14(28)$ & 0.29 \\
Low bicarbonate & $4(4.44)$ & $0(0)$ & 0.13 \\
Raised bicarbonate & $21(23.33)$ & $0(0)$ & 0.31
\end{tabular}

Note: *Significant; Chi-square test was done 
Serum bicarbonate level was significantly lower in the group with diabetes mellitus than in the control group $(27.90 \pm 3.44$ vs $28.20 \pm 2.10 ; \mathrm{P}=0.01)$. Although the level of serum

sodium and chloride were lower in group 1 , no significant variation was found compared to group 2 (Table 3 ). The mean anion gap of the cases with diabetes mellitus was normal $(13.92 \mathrm{mmol} / \mathrm{L})$.

There were significantly greater number of cases with hyponatraemia $(11.11 \%$ vs $0 \% ; \quad \mathrm{P}=0.01)$ and hypochloraemia $(48.89 \%$ vs $28 \%$; $\mathrm{P}=0.02)$ in the group with diabetes mellitus, compared with the group without diabetes mellitus There was no difference in other electrolytes abnormalities between the two groups (Table 4).

Table 5 Correlation of serum electrolytes with HbA1c, duration of diabetes and eGFR in
In patients with diabetes mellitus, there was no difference in electrolyte levels between those with and without hypertension. However, patients taking insulin had significantly lower bicarbonate level than those on lifestyle modification or oral medication $(24.92 \pm 3.86$ vs $28.35 \pm$ $2.95, \mathrm{P}<0.001)$.

Correlation of electrolytes with control, duration of diabetes and eGFR in patients with diabetes mellitus

Serum sodium was negatively correlated with $\mathrm{HbA1c}(\mathrm{r}=$ $0.39 ; \mathrm{P}<0.001)$ and duration $(\mathrm{r}=-0.31 ; \mathrm{P}=0.003)$ of diabetes. There was no significant correlation between other serum electrolytes and diabetes control and duration. There was no significant correlation between serum electrolytes and eGFR (Table 5). In linear regression analysis, serum sodium showed linear relation with $\mathrm{HbA} 1 \mathrm{c}$. When HbA1c increases by 1 unit, serum sodium will decrease by 0.62 units $(\beta=-0.62, P<0.001)$.

\section{patients with diabetes mellitus/group $1(\mathrm{n}=87)$}

\begin{tabular}{|c|c|c|c|c|c|c|}
\hline & \multicolumn{2}{|l|}{ HbA1c } & \multicolumn{2}{|c|}{$\begin{array}{l}\text { Duration of diabetes } \\
\text { mellitus }\end{array}$} & \multicolumn{2}{|l|}{ eGFR } \\
\hline & $\mathbf{r}$ & $\mathbf{P}$ & $\mathbf{r}$ & $\mathbf{P}$ & $\mathbf{r}$ & $\mathbf{P}$ \\
\hline Serum sodium & -0.39 & $<0.001^{*}$ & -0.31 & $0.003^{*}$ & -0.09 & 0.36 \\
\hline Serum potassium & 0.08 & 0.47 & 0.07 & 0.51 & -0.12 & 0.26 \\
\hline Serum chloride & 0.17 & 0.11 & -0.13 & 0.22 & -0.12 & 0.22 \\
\hline Serum bicarbonate & -0.17 & 0.11 & -0.06 & 0.55 & -0.09 & 0.36 \\
\hline
\end{tabular}

\footnotetext{
*Significant; Pearson's correlation was done
} 


\section{Discussion}

The present study showed that hyponatraemia and hypochloraemiawere more common in those with diabetes mellitus. Serum bicarbonate was significantly lower in group 1. Serum sodium decreased with increasing $\mathrm{HbA1c}$ and duration of diabetes.

Patients with diabetes mellitus had significantly lower levels of serum bicarbonate. Similar to our finding, another study also found low bicarbonate level in patients with diabetes mellitus (5). We were unable to do arterial blood gas analysis, however, none of the cases in the study were acutely ill or on ACEI. Therefore, insulin deficiency leading to ketoacidosis does not explain the fall of bicarbonate in these patients (13). The low bicarbonate level may be a result of proximal tubular loss of bicarbonate or severe insulin deficiency.

There were a significantly greater number of cases with hyponatraemia and hypochloraemia in the group with diabetes mellitus. Although some studies also reported a significant reduction in serum potassium, sodium and bicarbonate $(5,7)$ others found raised sodium and chloride levels in patients with diabetes mellitus (2,8.) However, comparison is difficult as not all these studies were done in people with normal renal function. The reduction may have been the result of electrolyte loss caused by kidney dysfunction. A limitation of this study is that 24-hour urine electrolytes were not determined.

Patients on insulin were found to have lower bicarbonate level. Insulin modulates the renal handling of different electrolytes without affecting the glomerular filtration rate. In contrast to this finding, there was an increase in urinary calcium, magnesium and phosphate excretion but a fall in potassium excretion in another study of renal handling of insulin ${ }^{(18)}$.
Insulin increases the permeability of cells to potassium, magnesium and phosphate ions causing a influx of these ions into cells. Insulin also activates sodium-potassium Insulin increases the permeability of cells to potassium, magnesium and phosphate ions causing a influx of these ions into cells. Insulin also activates sodium-potassium ATPases in many cells. $\mathrm{Na}+-\mathrm{K}+$-ATPase is thought to be linked to several complications of diabetes mellitus, hypertension and nephropathy ${ }^{(19)}$.

Serum sodium level decreased with poor glycaemic control and increasing duration of diabetes. However, there was no significant correlation with eGFR. Similarly, others found a significant association $(\mathrm{p}<0.001)$ between serum sodium levels and the fasting blood glucose levels (7). This may be explained by the fact that renal tubular function deteriorates with uncontrolled diabetes of prolong duration. Therefore there is increasing loss of sodium ion in the urine. Therefore, special attention should be paid to tubular function in diabetes mellitus. The estimated glomerular filtration rate (eGFR) alone is not sufficient to monitor renal function.

\section{Conclusion}

Electrolyte abnormalities are more common in patients with diabetes mellitus, even with normal creatinine and eGFR, and it is related to control and duration of diabetes. Diabetes mellitus patients with normal serum creatinine suffer from electrolyte abnormalities and should be investigated early in the course of disease.

Acknowledgements:

None.

Funding

None 


\section{References}

1. Marshall SM, Flyvbjerg A. Diabetic Nephropathy. In: Holt R I G, Cockram C S, Flyvbjerg A, Goldstein B J, editors. Textbook of Diabetes. 4th ed. Singapore: Wiley Blackwell; 2010. p. 599-614.

2. Sotirakopoulos N, Kalogiannidou I, Tersi M, Armentzioiou K, Sivridis D, Mavromatidis K. Acid-base and electrolyte disorders in patients with diabetes mellitus. Saudi J Kidney Dis Transpl. 2012; 23:58-62.

3. Shahid SM, Mahboob T. Electrolytes and Na+-K+-ATPase: potential risk factors for the development of diabetic nephropathy. Pak J Pharm Sci.2008; 21(2): 172-9.

4. Bukonla A, Benson OK, Akinsola AR, Aribigbola C, Adesola A, Seyi A. Effect of Type 1 Diabetes On Serum Electrolytes (Sodium and Potassium) Levels and Testosterone Hormone in Human Male Subjects. WebmedCentral Biochemistry.2012;3(9): WMC003698

5. Oyewole OI, Sessie S, Mansaray M, Kamara B. Changes in Serum Electrolytes and Lipid Profile in Diabetes Subjects in Freetown Sierra Leone. Sudan Journal of Medical Sciences.2008;3(4): 309-314.

6. Rao GM. Serum electrolytes and osmolality in diabetes mellitus. Indian J Med Sci.1992; 46 (10): 301-3.

7. Rubeaan K, Siddiqui K, Abu Risheh K, Hamsirani R, Alzekri A, Alaseem, A, Saleh SM, Al-Yami Z, Al -Ghamdia A, Alayed K. Correlation between Serum Electrolytes and Fasting Glucose and Hb1Ac in Saudi Diabetic Patients. Biol Trace Elem Res. 2011; 144(1-3): 463-8.

8. Datchinamoorthi S, Vanaja R, Rajagopalan B. Evaluation of Serum Electrolytes in Type 2 Diabetes Mellitus. Int. J. Pharm. Sci. Rev. Res. 2016; 40(1): 251-3

9. Anago EAA, Modehouenou TCM, Akpovi CD, Tchehouenou H. Electrolyte disturbances in diabetic patients in Cotonou, Benin. Int J Res Med Sci. 2016 ; 4(12): 5430-35

10. Shahid SM, Rafique R, Mahboob T. Electrolytes and sodium transport mechanism in diabetes mellitus. PakJ Pharm Sci. 2005; 18(2): 6-10.

11. Khan LA, Alam A M, Ali L, Goswami A, Hassan Z, Sattar S, Banik NG, Khan AK. Serum and urinary magnesium in young diabetic subjects in Bangladesh. Am J Clin Nutr. 1999; 69(1): 70-3.

12. Van Style DD, Serdroy J. Studies on diabetes and electrolyte equilibrium in blood. J. Biol.Chem.1928; 18: 20-26.

13. Haffner SM, Miettinen H. Insulin resistance implications for type II diabetes mellitus and coronary heart disease. Am J of Med.1997; 103(2): 152-62.

14. Leonard IK, John M. Physiology and pathophysiology of body water and electrolytes. In: Kaplan and Pesce, editors. Clinical Chemistry: Theory, Analysis and Correlation. The C.V. Mosby Co; 1989.

15. Liamis G, Liberopoulos E, Barkas F, Elisaf M. Diabetes Mellitus and Electrolyte disorders. World J Clin Cases. 2014; 2(10): 488-496.

16. Somogyi J, Kiss G, Pentek E, Csermely P. Diabetes mellitus as a general membrane disease and its consequences. Orv. Hetil. 2001; 142 (33):1781-8.

17. Saito T, Ishikawa S, Higashiyama M, Rokkaku K, Hayashi H, Kusaka I, Nagasaka S, Saito T. Inverse distribution of sodium and potassium in uncontrolled inpatients with diabetes mellitus. Endocr J.1999; 46(1): 75-80.

18. Rattanatayarom,W, Dorfmeisters C, Classen UG, Schimatschek HF, Stein U, Classen HG. Mg++ deficiency induced anorexia in hyperphagic obese zucker rats. Magnes Res. 2001; 14(3):181-8.

19. Ocsényi, Z., Tulassay, T., Miltényi,M., Szabó, A Effects of insulin on renal electrolyte handling. Child Nephrology and Urology. 1988 -1989; 9: 16-20. 